Relations industrielles

Industrial Relations

\title{
Comparative Studies in Organizational Behavior, by William K. Graham and Karlene H. Roberts, Editors, New York, Holt, Rinehart and Winston, Inc., 1972, 536 pp.
}

\section{Laurent Bélanger}

\section{Volume 29, numéro 4, 1974}

URI : https://id.erudit.org/iderudit/028573ar

DOI : https://doi.org/10.7202/028573ar

Aller au sommaire du numéro

Éditeur(s)

Département des relations industrielles de l'Université Laval

ISSN

0034-379X (imprimé)

1703-8138 (numérique)

Découvrir la revue

Citer ce compte rendu

Bélanger, L. (1974). Compte rendu de [Comparative Studies in Organizational Behavior, by William K. Graham and Karlene H. Roberts, Editors, New York, Holt, Rinehart and Winston, Inc., 1972, 536 pp.] Relations industrielles / Industrial Relations, 29(4), 892-893. https://doi.org/10.7202/028573ar

Tous droits réservés (C) Département des relations industrielles de l'Université Laval, 1974
Ce document est protégé par la loi sur le droit d'auteur. L’utilisation des services d’Érudit (y compris la reproduction) est assujettie à sa politique d'utilisation que vous pouvez consulter en ligne.

https://apropos.erudit.org/fr/usagers/politique-dutilisation/ 
loppement, celui de l'éducation formelle vue comme une industrie soulevant des problèmes particuliers quant aux résultats (outputs) du système, quant à l'accès à l'éducation, quant à l'orientation de différents niveaux d'éducation, quant à l'allocation de ressources financières et quant aux contraintes humaines et organisationnelles.

C'est au chapitre 4 qu'Harbison s'attaque d'une façon plus systématique à l'éducation informelle et la formation où il en identifie les fonctions et en donne quelques exemples.

La principale thèse développée sur ce sujet de l'apprentissage est que le développement efficace des ressources humaines exige l'intégration des processus d'éducation formels et informels durant la vie de toute personne. Son approche s'apparente grandement à celle de la formation continue.

La balance des cerveaux est équivalente pour Harbison à la balance commerciale de la balance des paiements i.e. l'importation (brain gain) et l'exportation de cerveaux (brain drain). Cette balance constitue selon lui un facteur stratégique important du développement national et c'est à ce problème qu'il consacre son cinquième chapitre.

Suite à l'identification de ces quatre problèmes majeurs de développement, Harbison s'attaque au $6 \mathrm{e}$ chapitre au développement national vu dans une perspective de ressources humaines. Il réalise cependant que son approche est une des approches possibles. Cependant il veut en examiner toutes les facettes. Ce n'est qu'au chapitre 7 que l'auteur s'attarde aux problèmes d'implantation de son approche.

En conclusion, l'auteur rappelle qu'il veut se dissocier de l'approche proprement économique pour en adopter une dite de ressources humaines. Sa principale thèse est que le développement des ressources en général ne peut se faire sans le développement des ressources humaines. Pour lui, c'est la base de la stratégie efficace du développement.

En résumé, il devient clair que cet ouvrage n'ajoute pas beaucoup à ce que Harbison a déjà écrit sur le sujet. Il semble que ce soit fondamentalement le même plat servi autrement. Plusieurs des critiques déjà formulées ne semblent pas avoir été prises en considération par l'auteur en écrivant son «nouveau» livre.

\section{Université Laval}

\section{Jean SEXTON}

Comparative Studies in Organizational Behavior, by William K. Graham and Karlene H. Roberts, Editors, New York, Holt, Rinehart and Winston, Inc., 1972, $536 \mathrm{pp}$.

Cet ouvrage qui traite de la psychosociologie des organisations se différencie des autres uniquement par la perspective que les deux éditeurs ont adoptée. De fait, au cours de la dernière décennie, on a assisté à la publication de nombreux travaux de recherche qui tentent de décrire et d'expliquer les différences au sein des organisations et entre les organisations au plan des structures et des modes d'adaptation des individus. Le volume est un recueil d'articles regroupés sous trois rubriques principales : a) les différences entre les niveaux et unités administratives au sein d'une organisation; b) les différences et les similitudes entre les organisations au sein d'un même contexte socio-culturel; c) les différences et les similitudes entre des organisations qui ouvrent dans des contextes socio-culturels différents.

Comme points de repère pour discuter des différences et des similitudes, les éditeurs ont retenu d'abord la motivation et la satisfaction au travail de même que les variables qui s'y rattachent. Ensuite, ils ont opté pour tous les articles importants et récents qui traitent des styles de leadership et de contrôle. Enfin, comme dernier point de repère, ils ont choisi des articles qui traitent de l'efficacité des organisations.

La plupart des articles qui apparaissent dans l'ouvrage ont été publiés dans des revues spécialisées au cours des années 60 . Ils sont déjà connus des lecteurs qui lisent régulièrement ces revues. Cependant, pour lire avec profit cet ouvrage, le lecteur doit considérer d'abord l'ensemble des articles qui apparaissent sous une même rubrique, aligner toutes les conclusions qui se dégagent à l'aide d'une grille quelconque et procéder à des comparaisoris, tout cela pour arriver à saisir l'apport des 
différents auteurs de même que les différences et similitudes. Le lecteur doit donc faire un effort additionnel pour mieux saisir la portée de tous ces travaux effectués à partir de cadres de référence particuliers à chacun des auteurs.

Sans doute, l'introduction rédigée par les éditeurs peut aider mais ce n'est pas suffisant. Ces derniers n'ont pas jugé bon de procéder à une réflexion d'ensemble qui aurait pu servir de conclusion à leur ouvrage.

\section{Laurent BELANGER}

Université Laval

Changement planifié et développement des organisations, sous la direction de Roger Tessier et Yvan Tellier, Paris, et Montréal, Edition conjointe EPIIFG, 1973, 825 p.

C'est beaucoup plus une présentation générale de cet ouvrage que nous voulons faire ici qu'une revue critique de chacun des articles écrits par différents auteurs dont la plupart sont québécois. L'ouvrage est un recueil de trente-sept articles auxquels on ajoute le compterendu d'une table ronde. Ces articles sont regroupés sous quatre grandes dimensions qui constituent les parties du recueil : la théorie du changement planifié et du développement des organisations; la pratique du changement planifié; le rôle de la recherche appliquée dans ce domaine; l'évaluation critique des fondements conceptuels qui soustendent la théorie et la pratique de l'intervention psychosociologique.

Dans l'introduction à leur recueil, les auteurs prennent soin de distinguer entre «changement planifié » au sens large et «changement planifié 》 au sens restreint du terme. La première signification regroupe tous les efforts systématiques de transformation sociale à l'échelle d'une société ou d'une communauté; la seconde origine du courant américain de la recherche-action et de la dynamique de groupe. Elle se confond actuellement avec le courant récent qu'on a appelé le D.O. ou «développement des organisations ». $\mathrm{Si}$ ce courant porte le nom de «planned changed $>$ ou changement planifié, il faut souligner que le changement ne se conçoit pas et ne s'implante pas de fa- çon autoritaire ou unilatérale comme dans le cas des changements pensés et conçus par des comités de planificateurs qu'on retrouve dans différents organismes de grande envergure ou de comités composés de technocrates au service des gouvernements. Au contraire, le développement des organisations (changement planifié, intervention psychosociologique) s'effectue sous un mode démocratique ou participatif, en impliquant les gens concernés dans le diagnostic de leurs problèmes et l'élaboration des changements à apporter.

Comme il n'existe pas de théorie générale du changement planifié, les auteurs parlent plutôt d'orientations théoriques dans la présentation de la première partie de l'ouvrage.

Sous ces orientations théoriques, on retrouve d'abord un essai de classification des expériences de changement planifié. Un second article traite du «sens de la complexité dans les organisations ». Dans un troisième, l'auteur, Roger Tessier, dresse «un tableau d'une stratégie de changement susceptible d'accroître le rendement de l'organisation bureaucratique dans une société en changement $\gg$ (p. 12). L'article de Yvan Tellier reprend le traditionnel débat sur le conflit 《line-staff » en l'analysant sous l'angle des rapports entre le pouvoir et le savoir. Richard Bekkard, un auteur américain, reprend le thème du malaise des cadres au sein des grandes organisations et du changement dans les systèmes de valeurs propres à cette catégorie. François Allaire traite du groupe de travail en effectuant une analogie entre ce dernier et les organisations. Dans «Experiencing et changement social», Jean Garneau essaie de démontrer qu'il est possible «de transposer au niveau $\mathrm{du}$ fonctionnement des groupes, les constatations faites au plan individuel sur les conditions favorisant le mouvement au sein du processus d'expériencing individuel $\gg$ (p. 13).

D'autres articles, dans cette même partie, reprennent le thème des stratégies et des tactiques de changement; des conditions d'implantation des changements; enfin, des modes d'influence qui peuvent s'établir entre les agents de changement et les systèmes-clients.

Les articles qui nous apparaissent les plus pertinents à une description des 